\title{
TACKLE MORE COMPLEX IMPLANT CASES
}

Hands-on training to enable experienced dental implant practitioners to tackle more complex cases takes place on 20-21 June 2013 at Advance Implant Clinic, Chelmsford. Presented by Dr Andrew Moore, the Advanced Implantology Course helps delegates to feel more confident managing bone deficiencies and undertaking immediate implant cases.

Dr Moore's course is designed for dentists who are already placing dental implants and wish to increase their clinical experience. The four live surgical sessions feature imme- diate placement and immediate loading. They demonstrate the restoration of the full arch using the ANKYLOS SynCone and SmartFix systems.

The course is approved by FGDP (UK) for accreditation of CPD hours and complies with GDC verifiable CPD requirements. Advance Implant Clinic is a purpose-built centre including state-of-the-art treatment facilities and a dedicated seminar room with surgery live on screen.

Further details can be found on the DENTSPLY Implants education website, www.courses4implants.com.

\section{EASILY CLEAN AND LUBRICATE YOUR HANDPIECES}

The Assistina 3X3 from W\&H is the ideal device for internal and external cleaning and lubrication of all handpieces.

The Assistina 3X3 cleans and lubricates three handpieces in three steps. Assistina combines thorough cleaning of internal spray channels and transmission parts with external handpiece cleaning and precise, consistent automatic lubrication of all internal components.

The Assistina 3X3 is validated to remove commonly encountered microbes in the dental surgery, with a cleaning efficacy of over 99\%. Using the Assistina 3X3 offers a real alternative to putting handpieces in a thermo washer disinfector, with gentle yet effective cleaning at ambient temperatures, using water-based active fluid designed especially for handpiece care. It prepares your handpieces quickly and easily prior to sterilisation.

In only 6.5 minutes you can ensure improved patient and user safety with the Assistina 3X3.

For a light hearted video which shows the Assistina 3X3 cleaning process go to www.youtube. com/user/ whdentalwerk and view: WetH Assistina 3x3 (English).

For further information, contact WetH (UK) Ltd on 01727 874990 or marketing.uk@wh.com.

\section{A FAST TRACK TO THE DIGITAL ORTHODONTICS HIGHWAY}

Stratasys, a leading manufacturer of 3D printers and production systems for prototyping and manufacturing, has announced its collaboration with Scheu Dental GmbH for offering orthodontic labs a complete digital solution.

Digital orthodontics is rapidly becoming the industry standard because of its many business advantages including the ability to sig- nificantly shorten delivery times, increase production capacity and eliminate bulky model storage.

According to Scheu Dental GmbH, the successful cooperation between the two companies in the orthodontic market will allow for a faster collaboration of the 'digital orthodontics highway' for all orthodontic labs worldwide, bringing mass customisation to small and mid-sized labs alike.

To find out more, visit www.stratasysdental.com. 